Portland State University

PDXScholar

$2-1-2000$

\title{
Effect of Slow Compression on the Linear Stability of an Accelerated Shear Layer
}

John D. Ramshaw

Portland State University, jdramshaw@yahoo.com

Follow this and additional works at: https://pdxscholar.library.pdx.edu/phy_fac

Part of the Fluid Dynamics Commons

Let us know how access to this document benefits you.

Citation Details

J.D. Ramshaw, "Effect of slow compression on the linear stability of an accelerated shear layer," Phys. Rev. E 61, 1486 (2000); erratum ibid. 64, 069904 (2001).

This Article is brought to you for free and open access. It has been accepted for inclusion in Physics Faculty Publications and Presentations by an authorized administrator of PDXScholar. Please contact us if we can make this document more accessible: pdxscholar@pdx.edu. 


\title{
Effect of slow compression on the linear stability of an accelerated shear layer
}

\author{
John D. Ramshaw \\ Lawrence Livermore National Laboratory, University of California, P.O. Box 808, L-097, Livermore, California 94551
}

(Received 28 May 1999)

\begin{abstract}
An analysis is given of the effect of a slow uniform anisotropic compression or expansion on the linear stability of a normally accelerated planar interface between two fluids with different densities and tangential velocities, i.e., a combined Kelvin-Helmholtz and Rayleigh-Taylor instability, but generalized to an arbitrary time-dependent acceleration history. The compression is presumed to be sufficiently slow that the density remains uniform within each fluid and hence depends only on time. The perturbation is taken to be sinusoidal with amplitude $h(t)$. The time evolution of $h$ is determined by requiring pressure continuity across the interface in the usual way. The resulting linearized stability equation is a second-order linear ordinary differential equation for $h(t)$. Compared to the corresponding well-known result for incompressible fluids, it is found that normal compression has the effect of reducing the perturbation growth rate $\dot{h}$ by an obvious geometrical correction, while transverse compression does not directly affect the net growth rate but rather has the dynamical effect of increasing its time derivative. When attention is focused on the masses transported across the initial interface rather than $h$, the purely geometrical effects of compression no longer appear explicitly, while the dynamical effects remain. It is thereby shown that both normal and transverse compression dynamically enhance the mixing of material masses, in spite of the corresponding purely geometrical reduction in $\dot{h}$.

PACS number(s): 47.20.Bp, 47.20.Ft, 47.20.Ma
\end{abstract}

\section{INTRODUCTION}

Linear stability analysis [1-3] remains an indispensable tool in the continuing effort to understand mixing at unstable fluid interfaces. The linear analysis provides an accurate description of the early stages of mixing, where the perturbation amplitude is small, and yields valuable analytical expressions for linear growth rates and their functional dependence on the various relevant parameters such as wavelength, gravity, density ratio, etc. The linear theory also serves as a cornerstone for the development of approximate theories and models to describe mixing at late times, where the dynamics becomes strongly nonlinear [4,5]. This was indeed the motivation for the present work, and a corresponding nonlinear model based on the present results will be described elsewhere. However, the linear case is also of intrinsic interest in its own right, as it provides a theoretical framework for interpreting the early-time dynamics of unstable fluid interfaces.

There are three classical interfacial instabilities, namely the Rayleigh-Taylor (RT) [2,3,6], Richtmyer-Meshkov (RM) $[7,8]$, and Kelvin-Helmholtz (KH) [1-3] instabilities. The linear theory of these instabilities in incompressible fluids is well known and well understood, both when they occur separately and when they occur together in various hybrid combinations. Indeed, a linear stability analysis is readily performed for a planar shear layer subjected to an arbitrary time-dependent acceleration in the normal direction [1-3], a situation which encompasses the three basic instabilities above as special cases. The analysis shows that the time evolution of the amplitude $h$ of a small sinusoidal perturbation with wavelength $\lambda=2 \pi / k$ is determined by the equation

$$
2 \bar{\rho} \ddot{h}=\left[\Delta \rho a k+\rho_{12}\left(k_{x} \Delta u\right)^{2}\right] h,
$$

where $\dot{q}=d q / d t, \rho_{i}$ is the density of fluid $i, 2 \bar{\rho}=\rho_{1}+\rho_{2}$, $\Delta \rho=\rho_{2}-\rho_{1}, \rho_{12}=\rho_{1} \rho_{2} /\left(\rho_{1}+\rho_{2}\right), u_{i}^{0}$ is the unperturbed tangential velocity of fluid $i$ parallel to the interface, $\Delta u$ $=u_{2}^{0}-u_{1}^{0}$ is the tangential velocity discontinuity, $k_{x}$ is the component of the perturbation wave vector along the direction associated with $\Delta u$, and the acceleration $a(t)$ is considered positive when directed from fluid 1 into fluid 2 . The same analysis further shows that the perturbation propagates along the interface with a phase velocity of

$$
\bar{u}_{0}=\frac{\rho_{1} u_{1}^{0}+\rho_{2} u_{2}^{0}}{\rho_{1}+\rho_{2}} .
$$

Equation (1) is valid only for $h \ll \lambda$, and is restricted to incompressible fluids for which $\rho_{i}$ is constant in space and time. In the absence of a better alternative, it has sometimes been employed as an approximation to compressible fluids as well, particularly at low to moderate Mach numbers. However, even when the Mach number is so low that the inhomogeneities associated with acoustic waves are negligible, compression or expansion of the mixing layer as a whole produces global effects that Eq. (1) does not capture, including the purely geometrical changes in $h$ and/or $k$ associated with compression or expansion in the normal and/or tangential directions, respectively. The correction for these geometrical effects is not however obvious, as they interact with and alter the dynamics. Our purpose here is to derive a generalization of Eq. (1) that consistently includes the effects of a slow uniform anisotropic compression or expansion of the mixing layer as a whole. Attention is restricted to compression and expansion rates which are sufficiently slow that the fluid densities $\rho_{i}$ remain uniform in space, and hence depend only upon time. The analysis therefore makes no attempt to 
represent the more complicated compressibility effects associated with nonzero Mach number and acoustic waves.

The derivation is based on potential flow theory. In Sec. II we obtain the linearized potential flow solution for a sinusoidal perturbation of an accelerated interface between two fluids with different densities and tangential velocities subjected to an externally imposed uniform but anisotropic compression or expansion. The corresponding linearized equation of motion for the perturbation amplitude $h$ is then derived in Sec. III by the conventional technique of requiring the pressure to be continuous across the interface. This then yields the desired compressive generalization of Eq. (1).

In the present context, however, the evolution equation for $h$ is not the whole story. The densities and interfacial area also change with time due to the compression, so that the perturbation amplitude $h$ alone no longer provides a suitable measure of the degree of mixing. It is therefore of interest to convert the evolution equation for $h$ into a corresponding evolution equation for the actual material masses $M_{i}$ transported across the unperturbed interface, which are a more accurate measure of the degree to which the two fluids have been mixed together by the instability. When this is done, the purely geometrical effects of the compression no longer appear explicitly, and only the dynamical effects remain. The resulting evolution equation for $M_{i}$ shows that compression dynamically enhances the mixing of material masses.

It is also of interest to compare the evolution equation for $M_{i}$ in the special case $\Delta u=0$ with that for the volume transported across a spherical interface between two concentric incompressible fluid shells [5]. These two equations are found to be identical in form when the compression rates are properly specialized to the spherical case. Finally, in Sec. IV we derive approximate analytical expressions for the growth rates of both $h$ and $M_{i}$ in the special case of constant normal acceleration.

\section{LINEARIZED POTENTIAL FLOW SOLUTION}

We consider an initially planar interface which separates two immiscible fluids with negligible surface tension in zero gravity. The unperturbed velocity of fluid $i$ is denoted by $\mathbf{u}_{i}^{0}$, which is presumed to be uniform and purely tangential to the interface. Thus $\mathbf{u}_{i}^{0} \cdot \mathbf{n}=0$, where $\mathbf{n}$ is the unit normal to the interface which points from fluid 1 into fluid 2 . The system is also subjected to a normal acceleration $a(t) \mathbf{n}$ relative to an inertial laboratory frame. It is convenient to describe the system in a Cartesian coordinate frame with the same acceleration. In this frame the system experiences an artificial external body force per unit mass of $-a(t) \mathbf{n}$, and the unperturbed interface is stationary for all $t$. The interface is then defined by the time-independent equation $\mathbf{n} \cdot \mathbf{r}=0$, where $\mathbf{r}$ is the position vector relative to an origin located somewhere on the interface. It is further convenient to let the coordinate frame move parallel to the plane of the interface with the velocity of the linear KH surface waves, so that these waves also become stationary. When this is done we have $\rho_{1} \mathbf{u}_{1}^{0}+\rho_{2} \mathbf{u}_{2}^{0}$ $=0$, which then implies

$$
\rho_{2} \mathbf{u}_{2}^{0}=-\rho_{1} \mathbf{u}_{1}^{0}=\rho_{12} \Delta \mathbf{u},
$$

where $\Delta \mathbf{u}=\mathbf{u}_{2}^{0}-\mathbf{u}_{1}^{0}$ is the tangential velocity discontinuity.
Without yet perturbing the interface, we now impose a uniform anisotropic compression upon the system, so that the unperturbed velocity of fluid $i$ becomes

$$
\mathbf{u}_{i}^{D}=\mathbf{u}_{i}^{0}+\mathrm{D} \cdot \mathbf{r},
$$

where the uniform symmetric tensor $\mathbf{D}$ is the gradient of the velocity field associated with the compression and expansion. Thus negative eigenvalues of $D$ imply compression, while positive eigenvalues imply expansion in their respective principal directions. In order to ensure that the compression and expansion do not change the normal direction $\mathbf{n}$, we must impose the condition $\mathbf{n} \cdot \mathbf{D} \cdot \mathbf{t}=0$, where $\mathbf{t}$ is any tangent vector normal to $\mathbf{n}$; i.e., $\mathbf{n} \cdot \mathbf{t}=0$. It follows that $D$ is of the form

$$
\mathrm{D}=D_{n} \mathbf{n n}+\mathrm{D}_{t},
$$

where $\mathrm{D}_{t} \cdot \mathbf{n}=0$. Since the compression and expansion are uniform, the fluid densities $\rho_{i}$ remain uniform within each fluid but now become dependent on time according to

$$
\dot{\rho}_{i}=-D \rho_{i},
$$

where $D=\boldsymbol{\nabla} \cdot \mathbf{u}_{i}^{D}=\mathrm{D}: \mathrm{U}$, and $\mathrm{U}$ is the unit dyadic.

We must of course require pressure continuity at the interface in the unperturbed solution, and this imposes some restrictions on $\mathbf{u}_{i}^{0}$ and $\mathbf{D}_{t}$. These restrictions may be inferred from the momentum equation of unperturbed fluid $i$,

$$
\rho_{i}\left(\frac{\partial \mathbf{u}_{i}^{D}}{\partial t}+\mathbf{u}_{i}^{D} \cdot \nabla \mathbf{u}_{i}^{D}\right)=-\nabla p_{i}^{D}-\rho_{i} a \mathbf{n},
$$

where $p_{i}^{D}$ is the unperturbed pressure in fluid $i$. Taking the dot product of the tangential projection tensor $\mathbf{U}-\mathbf{n n}$ into Eq. (7), combining the result with Eqs. (4) and (5), and evaluating the resulting equation on the interface where $\mathbf{r}$ becomes a purely tangential vector $\mathbf{t}$, we obtain

$$
\nabla_{t} p_{i}^{D}=-\rho_{i}\left[\dot{\mathbf{u}}_{i}^{0}+\mathrm{D}_{t} \cdot \mathbf{u}_{i}^{0}+\mathbf{t} \cdot\left(\dot{\mathrm{D}}_{t}+\mathrm{D}_{t} \cdot \mathrm{D}_{t}\right)\right],
$$

where $\boldsymbol{\nabla}_{t}=\boldsymbol{\nabla}-\mathbf{n n} \cdot \boldsymbol{\nabla}$ is the tangential gradient operator, and use has been made of the fact that $\mathbf{u}_{i}^{0} \cdot \mathbf{n}=0$. Pressure equality at the interface requires that $\nabla_{t} p_{1}^{D}=\nabla_{t} p_{2}^{D}$, and since the densities are generally different and $\mathbf{t}$ is arbitrary, this in turn implies

$$
\begin{aligned}
& \dot{\mathbf{u}}_{i}^{0}+\mathrm{D}_{t} \cdot \mathbf{u}_{i}^{0}=0, \\
& \dot{\mathrm{D}}_{t}+\mathrm{D}_{t} \cdot \mathrm{D}_{t}=0 .
\end{aligned}
$$

If Eqs. (9) and (10) are not satisfied, this merely implies that some other motion is also occurring in addition to the interfacial instabilities of present interest. In such cases, the unperturbed interface will no longer remain stationary but will undergo some additional distortion unrelated to the instability, and the present analysis will no longer rigorously apply. Even when Eqs. (9) and (10) are violated, however, interfacial instabilities will still occur, and the present analysis may still provide a useful description of these instabilities in situations where their growth rates are much larger than 
the characteristic frequencies associated with the underlying motions upon which they are superimposed.

We now perturb the interface by subjecting each point $\mathbf{r}$ thereof to a small vectorial displacement $h(t) C \mathbf{n}$, where $C$ $=\cos [\mathbf{k}(t) \cdot \mathbf{r}], \mathbf{k} \cdot \mathbf{n}=0$, and $|h \mathbf{k}| \ll 1$. The interface is now defined by the equation $\mathbf{n} \cdot \mathbf{r}=h C$. The time dependence of $\mathbf{k}$ is necessary to allow for the change in wavelength due to the tangential compression; i.e., nonzero $\mathrm{D}_{t}$. The time evolution of $\mathbf{k}$ may be determined by requiring the phase $\mathbf{k}(t) \cdot \mathbf{R}(t)$ to be independent of time for a point $\mathbf{R}(t)$ moving with the transverse compression velocity $\mathrm{D}_{t} \cdot \mathbf{R}$; i.e., $\dot{\mathbf{R}}=\mathrm{D}_{t} \cdot \mathbf{R}$. We thereby find

$$
\dot{\mathbf{k}}=-\mathrm{D}_{t} \cdot \mathbf{k}=-\mathrm{D} \cdot \mathbf{k} .
$$

We wish to evaluate the resulting potential flow field $\mathbf{u}_{i}$ $=\boldsymbol{\nabla} \phi_{i}$ in fluid $i$ to first order in $h$. The perturbation to the velocity field is $\mathbf{u}_{i}^{\prime} \equiv \mathbf{u}_{i}-\mathbf{u}_{i}^{D}$, and the perturbation to the potential is $\phi_{i}^{\prime} \equiv \phi_{i}-\phi_{i}^{D}$, where $\boldsymbol{\nabla} \phi_{i}^{D}=\mathbf{u}_{i}^{D}$ and $\boldsymbol{\nabla} \phi_{i}^{\prime}=\mathbf{u}_{i}^{\prime}$. Apart from irrelevant constants, the unperturbed velocity potentials are therefore given by

$$
\phi_{i}^{D}=\mathbf{u}_{i}^{0} \cdot \mathbf{r}+\frac{1}{2} \mathbf{r} \cdot \mathbf{D} \cdot \mathbf{r} .
$$

The externally imposed uniform compression is assumed to be sufficiently slow that acoustic effects can be neglected, so that $\boldsymbol{\nabla} \cdot \mathbf{u}_{i}$ remains uniform with the value $\mathrm{D}$. It then follows that $\boldsymbol{\nabla} \cdot \mathbf{u}_{i}^{\prime}=\nabla^{2} \phi_{i}^{\prime}=0$, so that the perturbation to the velocity potential remains harmonic even though $\nabla^{2} \phi_{i}=D \neq 0$. The boundary conditions on $\phi_{i}$ are (a) $\phi_{i} \rightarrow \phi_{i}^{D}$ (so that $\phi_{i}^{\prime}$ $\rightarrow 0$ ) far from the interface and (b) the normal derivative of $\phi_{i}$ on the interface must be the same as the normal velocity of the interface, with due allowance for the fact that the perturbation now causes the normal direction to differ slightly from $\mathbf{n}$. Condition (b) takes the form

$$
(\mathbf{n}+h S \mathbf{k}) \cdot \boldsymbol{\nabla} \phi_{i}=\dot{h} C-h S \dot{\mathbf{k}} \cdot \mathbf{r},
$$

where $S=\sin [\mathbf{k}(t) \cdot \mathbf{r}]$, and the derivatives are evaluated at $\mathbf{n} \cdot \mathbf{r}=h C$. In the linear approximation, Eq. (13) reduces to

$$
\left(\frac{\partial \phi_{i}^{\prime}}{\partial n}\right)_{\mathbf{n} \cdot \mathbf{r}=0}=\left(\dot{h}-D_{n} h\right) C-h S \mathbf{u}_{i}^{0} \cdot \mathbf{k},
$$

where $\partial \phi_{i}^{\prime} / \partial n \equiv \boldsymbol{\nabla} \phi_{i}^{\prime} \cdot \mathbf{n}$, and use has been made of Eq. (11). The solutions to Laplace's equation that satisfy Eq. (14) and vanish at infinity are readily found to be

$$
\phi_{i}^{\prime}=\mp \frac{1}{k}\left[\left(\dot{h}-D_{n} h\right) C-h S \mathbf{u}_{i}^{0} \cdot \mathbf{k}\right] e^{\mp k \cdot \mathbf{r}},
$$

where $k=|\mathbf{k}|$, the upper sign applies for $\mathbf{n} \cdot \mathbf{r}>h C(i=2)$, and the lower sign for $\mathbf{n} \cdot \mathbf{r}<h C(i=1)$. The velocity of fluid $i$ is then given by $\mathbf{u}_{i}=\mathbf{u}_{i}^{0}+\mathrm{D} \cdot \mathbf{r}+\boldsymbol{\nabla} \phi_{i}^{\prime}$.

\section{LINEARIZED EQUATION OF MOTION}

Equation (12) and (15) determine the potentials $\phi_{i}=\phi_{i}^{D}$ $+\phi_{i}^{\prime}$ and hence the velocities $\mathbf{u}_{i}=\mathbf{u}_{i}^{D}+\boldsymbol{\nabla} \phi_{i}^{\prime}$ resulting from a prescribed perturbation amplitude $h(t)$. For an arbitrary $h(t)$, however, the corresponding pressures in the two fluids will in general be discontinuous across the interface. The natural motion of the system is that which preserves pressure continuity at the interface $\mathbf{n} \cdot \mathbf{r}=h(t) C$, and this condition may be imposed to determine the linearized equation of motion for $h(t)$. For this purpose we require a suitable expression for the pressure $p_{i}$ in fluid $i$. If the flow were incompressible, $p_{i}$ could be obtained from the time-dependent Bernoulli equation [9]

$$
p_{i}=-\rho_{i}\left(\frac{\partial \phi_{i}}{\partial t}+\frac{1}{2}\left|\boldsymbol{\nabla} \phi_{i}\right|^{2}+a(t) \mathbf{n} \cdot \mathbf{r}\right)+F_{i}(t),
$$

where $F_{i}(t)$ is a function of time alone. In the present context the flow is not incompressible, and $\rho_{i}$ depends on time according to Eq. (6). Fortunately, however, it is easy to verify that Eq. (16) remains valid even in this case, provided that $\rho_{i}$ remains uniform as it does here. (This follows directly from the momentum equation in the usual way.) We may therefore employ Eq. (16) in the present context. To do so, we simply substitute $\phi_{i}=\phi_{i}^{D}+\phi_{i}^{\prime}$ into Eq. (16), evaluate the resulting $p_{i}$ at $\mathbf{n} \cdot \mathbf{r}=h(t) C$, and linearize in $h$. After some algebra, we thereby obtain

$$
p_{i}=p_{i}^{0}+p_{i}^{C} C+p_{i}^{S} S,
$$

where

$$
\begin{gathered}
p_{i}^{0}=-\frac{1}{2} \rho_{i}\left|\mathbf{u}_{i}^{0}\right|^{2}+F_{i}(t), \\
k p_{i}^{C}= \pm \rho_{i}\left[\frac{d}{d t}\left(\dot{h}-D_{n} h\right)+D_{k}\left(\dot{h}-D_{n} h\right)-\left(\mathbf{u}_{i}^{0} \cdot \mathbf{k}\right)^{2} h\right] \\
-\rho_{i} a k h, \\
k p_{i}^{S}=\mp \rho_{i} \mathbf{u}_{i}^{0} \cdot\left[\left(2 \dot{h}+D_{k} h-D_{n} h\right) \mathbf{k}-2 h \mathrm{D} \cdot \mathbf{k}\right]
\end{gathered}
$$

in which

$$
k^{2} D_{k}=\mathbf{k} \cdot \mathrm{D} \cdot \mathbf{k}=\mathbf{k} \cdot \mathrm{D}_{t} \cdot \mathbf{k}
$$

and use has been made of Eqs. (9)-(11).

The linearized equation of motion for $h$ is obtained by requiring $p_{1}=p_{2}$ for all $\mathbf{k} \cdot \mathbf{r}$. The three terms in $p_{i}$ are linearly independent, so their coefficients must be separately equal, i.e., $p_{1}^{0}=p_{2}^{0}, p_{1}^{S}=p_{2}^{S}$, and $p_{1}^{C}=p_{2}^{C}$. The first of these conditions provides no information, as it merely relates $F_{1}(t)$ and $F_{2}(t)$. (It does, however, have thermodynamic implications as discussed in Sec. V below.) The second condition reduces to an identity by virtue of Eq. (3), thereby confirming that Eq. (3) does indeed imply stationary $\mathrm{KH}$ surface waves even when compression is present. Thus the only nontrivial condition is $p_{1}^{C}=p_{2}^{C}$, which yields

$$
\frac{d}{d t}\left(\dot{h}-D_{n} h\right)+D_{k}\left(\dot{h}-D_{n} h\right)=\left[A k a(t)+B(\mathbf{k} \cdot \Delta \mathbf{u})^{2}\right] h,
$$

where $A=\left(\rho_{2}-\rho_{1}\right) /\left(\rho_{2}+\rho_{1}\right)=\Delta \rho /(2 \bar{\rho})$ is the Atwood number, $B=\rho_{1} \rho_{2} /\left(\rho_{1}+\rho_{2}\right)^{2}=\rho_{12} /(2 \bar{\rho})$, and use has been made of Eq. (3). 
Equation (22) is the desired generalization of Eq. (1) to allow for nonzero values of $D$. It is the linearized equation of motion for the perturbation amplitude in an accelerated shear layer with slow compression, and it properly reduces to the incompressible Eq. (1) when $\mathrm{D}=0$ as it should. Comparison with Eq. (1) shows that normal compression (negative $D_{n}$ ) has the obvious geometrical effect of reducing the perturbation growth rate by an amount $\left|D_{n}\right| h$. However, we show below that this reduction has no effect on the fluid masses transported across the interface or mixed together by the instability. It is entirely analogous to the purely geometrical effects on perturbation amplitude that occur in spherical geometry [5]. Comparison with Eq. (1) further shows that transverse compression (negative $D_{k}$ ) does not directly affect the net perturbation growth rate $\dot{h}-D_{n} h$ itself, but rather has the dynamical effect of increasing its time derivative by an amount $\left|D_{k}\right|\left(\dot{h}-D_{n} h\right)$.

It is of interest to derive a corresponding evolution equation for the mass $M_{i}(t)$ of fluid $i$ which has moved across some Lagrangian area $A$ of the original interface by time $t$. This mass is readily found to be $M_{i}(t)=\pi^{-1} \rho_{i} A h$, where $\dot{A}=\left(\mathrm{D}_{t}: \mathrm{U}\right) A$ due to the transverse compression. Eliminating $h$ and its derivatives from Eq. (22) in favor of $M_{i}(t)$ and its derivatives, we readily obtain

$$
\ddot{M}_{i}+\left(D_{n}+D_{k}\right) \dot{M}_{i}=\left[A k a(t)+B(\mathbf{k} \cdot \Delta \mathbf{u})^{2}\right] M_{i},
$$

where use has been made of Eq. (6). Comparison with Eq. (22) shows that the transformation to $M_{i}$ as a variable has eliminated the purely geometrical correction discussed above, as $\dot{M}_{i}$ itself is not modified by compressive effects. However, the dynamical effects of compression remain and are represented by the term $\left(D_{n}+D_{k}\right) \dot{M}_{i}$, which subtracts from $\ddot{M}_{i}$. For a growing perturbation with $M_{i}, \dot{M}_{i}>0$, compression $\left(D_{n}, D_{k}<0\right)$ therefore has the effect of increasing $\ddot{M}_{i}$. Thus we see that compression dynamically enhances mixing, whereas a superficial interpretation of the simultaneous but purely geometrical reduction in $\dot{h}$ might erroneously lead one to the opposite conclusion. The physical interpretation of this dynamical effect is not obvious in the present treatment, but it will be shown elsewhere that this effect represents the amplification of kinetic energy by compression, and hence is closely analogous to $p d V$ work.

It is also of interest to compare Eq. (23) with the corresponding growth law for an accelerated spherical interface of radius $R$ between concentric incompressible fluid shells [5], where the variable corresponding to $M_{i}$ is $s=R^{2} h$, which is proportional to the volume transported across the unperturbed spherical interface. In the limit of large perturbation mode number $\ell$, the evolution equation for $s$ takes the form $[5]$

$$
\ddot{s}-\frac{\dot{R}}{R} \dot{s}=A k a(t) s,
$$

where $k=\ell / R$ is the effective wave number of the perturbation [5]. Equation (24) is formally identical with Eq. (23) for $\Delta u=0$ if $D_{n}+D_{k}$ is identified with $-\dot{R} / R$. This identification is indeed appropriate, and this is easily established as follows. The unperturbed incompressible radial velocity field in spherical geometry is given by $u_{r}=R^{2} \dot{R} / r^{2}$, so that $D_{n}$ $=\partial u_{r} /\left.\partial r\right|_{r=R}=-2 \dot{R} / R$. Moreover, in spherical geometry the area $A$ is simply proportional to $R^{2}$, so that $\mathrm{D}_{t}: \mathrm{U}=\dot{A} / A$ $=2 \dot{R} / R$. But in this context any two orthogonal tangential directions are equivalent by symmetry, so that $D_{k}$ $=(1 / 2) \mathrm{D}_{t}: \mathrm{U}=\dot{R} / R$. Thus $D_{n}+D_{k}=-2 \dot{R} / R+\dot{R} / R=-\mathrm{R} / \mathrm{R}$. When properly interpreted, Eqs. (22) and (23) therefore also apply in spherical geometry as a special case. This is quite remarkable, and makes it tempting to speculate that these equations may provide a useful approximate description of compression and convergence effects on deforming curved interfaces in general, provided of course that the radius of curvature remains much larger than the amplitude and wavelength of the perturbations.

\section{APPROXIMATE GROWTH RATES FOR CONSTANT ACCELERATION}

When $a$ and $\Delta u$ are constant in time, Eq. (1) can of course be solved analytically, and one obtains exponential solutions (possibly complex) in the usual way. This is no longer the case for Eqs. (22) and (23), since $\Delta \mathbf{u}, \mathbf{k}, \mathrm{D}_{t}$, and possibly $D_{n}$ still depend on time according to Eqs. (9)-(11). However, it is of interest to consider the approximation in which these quantities are regarded as constant in time, which should be valid and useful in cases where they vary slowly compared to the growth rate of the instability. We therefore proceed to determine the growth rates of $h$ and $M_{i}$ for constant $a$ in this approximation.

Substituting $h=\alpha \exp \left(\gamma_{h} t\right)$ into Eq. (22) and solving for $\gamma_{h}$, we find that

$$
\gamma_{h}=\frac{1}{2}\left(D_{n}-D_{k}\right) \pm \sqrt{A k a+B(\mathbf{k} \cdot \Delta \mathbf{u})^{2}+\frac{1}{4}\left(D_{n}+D_{k}\right)^{2}} .
$$

Similarly, setting $M_{i}=\alpha \exp \left(\gamma_{M} t\right)$ in Eq. (23), we obtain

$$
\begin{aligned}
\gamma_{M} & =-\frac{1}{2}\left(D_{n}+D_{k}\right) \pm \sqrt{A k a+B(\mathbf{k} \cdot \Delta \mathbf{u})^{2}+\frac{1}{4}\left(D_{n}+D_{k}\right)^{2}} \\
& =\gamma_{h}-D_{n}
\end{aligned}
$$

which again exhibits the removal of the purely geometrical contribution of $D_{n}$ to the growth rate of $h$. These equations explicitly display the effects of $D$ on the growth rates. They are unlikely to find much quantitative application in practical situations, where $a$ is rarely constant, but they may nevertheless be useful in providing qualitative insight into the various functional dependences. For example, it is instructive to examine the effect of compression on a pure RM instability, for which $\Delta \mathbf{u}=0$ and $a(t)=\Delta \mathrm{v} \delta(t)$, so that $a(t)=0$ for $t>0$. Equation (25) then gives $\gamma_{h}=D_{n}$ and $\gamma_{h}=-D_{k}$, so that $h$ $=\alpha \exp \left(D_{n} t\right)+\beta \exp \left(-D_{k} t\right)$, where $\alpha$ and $\beta$ are determined by the initial conditions $h_{0} \equiv h(t=0)$ and $\dot{h}_{0} \equiv \dot{h}(t=0+)$, in terms of which we readily find $\alpha+\beta=h_{0}$ and $D_{n} \alpha-D_{k} \beta$ $=\dot{h}_{0}$. It follows from Eq. (22) that $\dot{h}(0+)=\dot{h}(0-)$ $+A k \Delta \mathrm{v} h_{0}$, and that $\dot{h}=D_{n} h$ for $t<0$, so that $\dot{h}(0-)$ $=D_{n} h_{0}$ and $\dot{h}(0+) \equiv \dot{h}_{0}=h_{0}\left(D_{n}+A k \Delta \mathrm{v}\right)$. These relations 
determine $\alpha$ and $\beta$ in terms of $h_{0}$ and $\Delta \mathrm{v}$. Thus a slow compression or expansion has the effect of changing the RM time dependence of $h$ from linear to exponential. When $\max \left(\left|D_{n}\right|,\left|D_{k}\right|\right) t \ll 1$, the exponentials can be linearized and we then regain the usual linearized RM growth law $h=h_{0}(1$ $+A k \Delta \mathrm{v} t)$. It would be of interest to verify the predicted exponential time dependence either experimentally (e.g., in a cylinder fitted with a piston) or by means of direct numerical simulations.

\section{CONCLUSION}

The effect of a slow uniform but anisotropic compression or expansion on the linear stability of an accelerated shear layer has been derived. The resulting equation of motion for the amplitude of a small sinusoidal perturbation is given by Eq. (22), while the corresponding evolution equation for the actual material masses transported across the original planar interface is given by Eq. (23). The latter equation shows that compression dynamically enhances the mixing of material masses, in spite of the associated but purely geometrical reduction in $\dot{h}$.

We have restricted attention to situations in which the velocity gradient $\mathrm{D}$ associated with the compression and expansion is uniform in all space and hence is the same in both fluids. It should be noted that this restriction is thermodynamically consistent only when both fluids have the same compressibility. The reason is that $p_{i}^{0}$ in Eq. (17) is not merely the uniform part of the dynamic pressure of Eq. (16), but also represents the thermodynamic pressure of fluid $i$, which is related to $\rho_{i}$ by an equation of state. For an isentropic compression, the condition $p_{1}^{0}=p_{2}^{0}$ then implies that $\rho_{1} c_{1}^{2} D_{1}=\rho_{2} c_{2}^{2} D_{2}$, where $c_{i}$ and $D_{i}$ are, respectively, the sound speed and velocity divergence in fluid $i$. Our restriction to a globally uniform $\mathrm{D}$ implies $D_{1}=D_{2}$, and is therefore tantamount to a restriction to cases in which the two fluids have the same compressibility; i.e., $\rho_{1} c_{1}^{2}=\rho_{2} c_{2}^{2}$. The generalization of the analysis to allow $D_{1} \neq D_{2}$ unfortunately presents nontrivial complications, which we hope to discuss elsewhere.

\section{ACKNOWLEDGMENTS}

I am grateful to Larry Cloutman and Karnig Mikaelian for many helpful discussions and for directing my attention to some of the relevant literature. This work was performed under the auspices of the U.S. Department of Energy by Lawrence Livermore National Laboratory under Contract No. W-7405-ENG-48.
[1] H. Lamb, Hydrodynamics, 6th ed. (Dover, New York, 1945).

[2] S. Chandrasekhar, Hydrodynamic and Hydromagnetic Stability (Dover, New York, 1981).

[3] P. G. Drazin and W. H. Reid, Hydrodynamic Stability (Cambridge University Press, Cambridge, 1981).

[4] J. D. Ramshaw, Phys. Rev. E 58, 5834 (1998).

[5] J. D. Ramshaw, Phys. Rev. E 60, 1775 (1999).
[6] H. J. Kull, Phys. Rep. 206, 197 (1991).

[7] R. D. Richtmyer, Commun. Pure Appl. Math. 13, 297 (1960).

[8] E. E. Meshkov, Izv. Akad. Nauk SSSR Mekh. Zhidk. Gaza 4, 151 (1969) [Izv. Acad. Sci. USSR Fluid Dyn. 4, 101 (1969)].

[9] G. K. Batchelor, An Introduction to Fluid Dynamics (Cambridge University Press, Cambridge, 1970). 